\title{
Los debates electorales de 2015 y 2016 en España. Contexto de percepción y credibilidad para los jóvenes
}

\author{
The electoral debates of 2015 and 2016 in Spain. Context of \\ perception and credibility for young people
}

\author{
Alfredo Arceo Vacas. Universidad Complutense de Madrid. España. \\ aarceo@ucm.es \\ $[\mathrm{CV}]$ (1) G $\mathrm{G}$ \\ Sergio Álvarez Sánchez. Universidad Complutense de Madrid. España. \\ sergioalvarezsanchez@ucm.es \\ $[\mathrm{CV}] \odot \mathrm{G} \mathrm{R}^{\mathrm{G}} \mathrm{G}$ \\ Olga Serrano Villalobos. Universidad Rey Juan Carlos. España. \\ olga.serrano@urjc.es \\ $[\underline{\mathrm{CV}}] \odot \mathrm{R}^{\mathrm{O}} \mathrm{O}$
}

Este artículo es producto del proyecto de investigación "Los debates electorales televisados durante las campañas del 20-D de 2015 y el 26-J de 2016: estudio comparativo de los mensajes y la imagen proyectada por los candidatos", referencia PR41/17-20954, financiado por el organismo público Universidad Complutense con el apoyo de la entidad privada Santander, dentro del programa de ayudas para la financiación de proyectos de investigación PR41/17.

Fechas:

- inicio de la investigación: 15 de diciembre de 2017.

- término de la investigación: 15 de diciembre de 2018.

\section{Agradecimiento}

Carlos Correa Paz

Socio y director de CeCubo Group, España, por el suministro de datos de Twitter mediante la herramienta CeCubo Metrics-carlos.correa@,cecubogroup.com

\section{Cómo citar este artículo / Referencia normalizada}

Arceo Vacas, A.; Álvarez Sánchez, S., y Serrano Villalobos, O. (2020). Los debates electorales de 2015 y 2016 en España. Contexto de percepción y credibilidad para los jóvenes. Revista Latina de Comunicación Social, (76), 17-37. https://www.doi.org/10.4185/RLCS-2020-1435

\section{RESUMEN}

Introducción: Se describe la influencia del contexto de percepción de los debates electorales del 14 de diciembre de 2015 y el 13 de junio de 2016, en la credibilidad atribuida a los candidatos, y el grado de entendimiento alcanzado. Metodología: Se revisaron encuestas de la época; se practicó un análisis descriptivo de noticias en prensa; se celebraron dos grupos de discusión con jóvenes, sobre los juicios de credibilidad; y se realizaron análisis de contenido a los mensajes en Twitter de los candidatos. Resultados: La prensa propició un contexto de emociones negativas; los candidatos destinaron Twitter a demostrar eficacia social, pero fracasaron entre el público joven. Discusión: 
Que no se percibiera el subfactor de credibilidad más trabajado, debería preocupar a los equipos de campaña. Conclusiones: Los candidatos habrían ganado credibilidad trabajando otros subfactores, en vez de centrarse sólo en la eficacia social; y mostrándose más naturales (algo que logró Rajoy).

PALABRAS CLAVE: comunicación política; elecciones; debates electorales; credibilidad; redes sociales; prensa.

\begin{abstract}
Introduction: This article describes the influence of the context of perception of the electoral debates broadcasted on 2015 December 14th and 2016 June 13th, in the credibility attributed to the candidates, and the reached degree of understanding. Method: Polls of that time were reviewed; a descriptive analysis of the press news was conducted; two focus groups on credibility judgments with young actuants were organized; and content analyses of the candidates' messages on Twitter were performed. Results: The press favored a context of negative emotions; the candidates used Twitter to show their social efficacy, but failed to convince young people. Discussion: Campaign teams should worry for the lack of perception of the credibility subfactor most worked by candidates. Conclusions: The candidates would have looked more credible had they worked on other credibility subfactors, instead of focusing only on social efficacy; and by acting more spontaneously (something on which Rajoy succeeded).
\end{abstract}

KEYWORDS: political communication; elections; electoral debates; credibility; social media; press.

\title{
CONTENIDOS
}

1. Introducción. 1.1. Propósito y relevancia de la investigación. 1.2. Relaciones públicas en la comunicación política. 1.3. El enmarcado de los mensajes electorales. 1.4. El estudio de la credibilidad percibida. 2. Objetivos. 3. Metodología. 3.1. Enfoque metodológico. 3.2. Revisión de encuestas electorales y de opinión. 3.3. Celebración de grupos de discusión. 3.4. Estudio descriptivo de las noticias de prensa. 3.5. Análisis de contenido de cuentas de Twitter. 4. Resultados. 4.1. Información relevante de las encuestas. 4.2. Tratamiento de las campañas en prensa. 4.3. Síntesis de los grupos de discusión. 4.4. Análisis de los perfiles en Twitter de los candidatos. 5. Discusión. 6. Conclusiones. 7. Referencias. 7.1. Artículos relacionados.

\section{Introducción}

La presente investigación tiene por objeto describir los contextos de percepción de dos debates electorales que marcaron un punto de inflexión en la comunicación política española: El encuentro cara a cara entre Mariano Rajoy (presidente en funciones en aquel momento y candidato del Partido Popular a la presidencia del Gobierno) y Pedro Sánchez (candidato del Partido Socialista), emitido el 14 de diciembre de 2015; y el debate a cuatro entre Mariano Rajoy, Pedro Sánchez, Pablo Iglesias (candidato de Podemos) y Albert Rivera (candidato de Ciudadanos), celebrado el 13 de junio de 2016.

El contexto sociopolítico en el que se producen los efectos de los medios sobre la audiencia es un factor a tener en cuenta para valorar sus consecuencias pues, sin ir más lejos, se han hallado diferencias entre el impacto de la dimensión afectiva de las noticias durante un periodo de campaña y fuera de éste (Aaldering, Van der Meer y Van der Brug, 2018, p. 87). En cada contexto específico, para alcanzar el éxito electoral, un candidato necesita convencer de sus capacidades a los votantes. En los últimos tiempos, esta necesidad ha dado lugar a una progresiva personalización de la política en sus líderes, bien sea mediante un mayor peso a éstos en comparación con la atención mediática a sus partidos; o bien mediante una mayor atención a la vida personal de los políticos, frente a su vida 
pública (Van Aelst, Sheafer y Stanyer, 2011, p. 214). A su vez, la personalización impacta en las dinámicas de la comunicación mediada en las campañas: Sólo cuando la sociedad se encuentra sumida en uno de estos periodos, se ha constatado cómo las imágenes positivas para la percepción del liderazgo de los candidatos tienen un mayor efecto en la intención de voto del público que las negativas (Aaldering et al., 2018, p. 86).

Sin embargo, existe un formato en el que los candidatos disfrutan de un menor grado de mediatización para poder presentar sus propuestas políticas a los votantes: El debate electoral (López-García, Llorca-Abad, Valera-Ordaz y Peris-Blanes, 2018, pp. 790-791). Por su parte, el público, gracias a esta particularidad, se sirve de los debates electorales televisados para detectar incoherencias entre los mensajes de los candidatos en estos formatos, y sus declaraciones de otras ocasiones (Coleman y Moss, 2016, p. 13).

Ahora bien, tanto los efectos de los debates electorales como los de los distintos contenidos mediáticos, se despliegan en un entorno en el que cada vez tiene un papel más prominente la comunicación digital. Sin embargo, los primeros indicios apuntan hacia una infrautilización de sus plataformas. El estudio de Chaves-Montero y Gadea (2017, p. 13) detectó que los partidos y candidatos tienden a conceder más relevancia a Twitter que a Facebook; y que ambos desaprovechan sus posibilidades para, mediante la interactuación, incrementar su credibilidad percibida. En la campaña de 2015, el 87,6 \% de las publicaciones en Facebook analizadas por Ballesteros $(2017$, p. 8) carecían de afán movilizador, destinándose el 35,4\% a la narración de experiencias; además, la dinamización de debates y las respuestas apenas llegaron al $1 \%$ de lo publicado. Sólo Pablo Iglesias generó debate en Facebook más allá de autopromocionarse (Chaves-Montero y Gadea, 2017, p. 17). También fueron excepciones Albert Rivera e Izquierda Unida por sus esfuerzos movilizadores, siendo la del PP la cuenta que menos llamaba a la acción (Ballesteros, 2017, p. 9). Aun así, casi todas las llamadas a la movilización en Facebook se reducían a la asistencia a mítines (Ballesteros, 2017, p. 14). De hecho, por lo que respecta al aprovechamiento de las redes sociales, tanto los candidatos como los partidos emplean Twitter fundamentalmente como herramienta de distribución de contenidos, en vez de como espacio para gestionar un debate con los públicos, en paralelo al que se esté televisando (Lozano Aguilar y García Orosa, 2017, pp. 237-238).

En suma, el problema que la presente investigación se propone resolver, se refiere a la determinación del impacto de los contextos en la percepción de los debates electorales por parte de los votantes. Los debates no se pueden estudiar aislándolos de la realidad descrita para la comunicación digital, ni de las especificidades que los propios candidatos y partidos generan con su comunicación durante las campañas. Para comprender las dinámicas y los efectos de los dos debates electorales seleccionados, resulta ineludible esbozar sus contextos de percepción. Al incorporar esta dimensión, se pone fin a una carencia habitual en los análisis. En este sentido, el trabajo de Aaldering et al. (2018) proporciona claves que evidencian la importancia de los contextos de percepción, y ha resultado un estudio fundamental para estimar que la presente investigación debe seguir la evolución de dichos contextos a lo largo de cada campaña.

Analizar esta serie de cuestiones (y, en particular, cómo impactaron los esfuerzos comunicativos de los candidatos en los contextos de percepción de los debates electorales de diciembre de 2015 y junio de 2016), es una labor especialmente relevante por la situación inédita en la historia política española, sin referencias previas. Emergían dos nuevos partidos que por primera vez desafiaban a PP y PSOE (Podemos en la izquierda y Ciudadanos como opción liberal), experimentándose un bloqueo político entre los dos comicios mencionados, por falta de acuerdo. En consecuencia, resulta pertinente el estudio atento y específico de los debates electorales de ambas campañas y sus contextos. 


\subsection{Relaciones públicas en la comunicación política}

Las relaciones públicas están presentes en la esfera política, como lo están en la pública y la privada. Son imprescindibles para que un candidato electoral alcance el entendimiento con sus públicos, a través del conocimiento de los asuntos que les preocupan y las expectativas que albergan. El concepto de relaciones públicas en el ámbito político se define como:

El proceso de gestión por el que una organización o actor individual, para unos propósitos

políticos y mediante una comunicación y acción orientadas a objetivos, persigue influir, así

como establecer, construir y mantener relaciones y reputaciones beneficiosas con sus públicos

clave para ayudarle a sostener su misión y alcanzar sus objetivos. (Strömback y Kiousis,

2011, p. 8)

Paralelamente, la comunicación política "limita con muchos territorios en los que conviven la politología, la sociología, la antropología, las ciencias de la comunicación y de la opinión pública, la psicología, la retórica y la publicidad" (Mazzoleni, 2010, p. 17). Si se atiende a ambas ideas, el campo de la comunicación política englobaría cualquier intercambio de mensajes sobre las decisiones que afectan colectivamente a una comunidad; por el contrario, para las relaciones públicas en la esfera política tienen que darse la construcción y cuidado de la relación, así como el mutuo beneficio entre el político y sus públicos. A la hora de emplear dimensiones con las que medir las estrategias de relaciones públicas políticas que se desplieguen, Seltzer y Zhang (2011, pp. 27-28) extraen cuatro variables de la literatura de la disciplina: El control mutuo (es decir, el grado de acuerdo acerca de quién está legitimado para ejercer el poder), la confianza, la satisfacción y la dedicación o compromiso.

En consecuencia, para analizar si esa bidireccionalidad se produce en una campaña de relaciones públicas en cualquier ámbito, Broom (1977) tomó el modelo de coorientación de McLeod y Chaffe (1973) como base para conceptualizar una serie de variables de relación de doble flujo (es decir, bidireccionales): comunicación (dimensión del dialogo), entendimiento (conocimiento compartido), acuerdo (actitudes compartidas) y congruencia (o comportamiento complementario). Junto a estas variables, en el modelo de coorientación, la precisión se refiere a la coincidencia entre lo que una parte cree que su interlocutor tiene en la cabeza, y lo que realmente piensa éste (Walden, Bortree y Di Stasso, 2015, p. 527). La precisión cobra una especial importancia en la comunicación política, porque implica a las orientaciones hacia el objeto (en el caso de esta investigación, un candidato electoral) de ambas partes de la relación (Eveland y Hutchens, 2013, p. 424).

Estas dimensiones implican que los juicios de los públicos sobre la persona u organización con la que se están relacionando, están en constante evolución por los efectos del posible entendimiento y acuerdo que se logre en cada momento. Por ello, el modelo de coorientación, en la adaptación que Broom (1977) realiza para el campo de las relaciones públicas invita, por una parte, a establecer objetivos orientados a hallar la evolución de los contextos y las estrategias a lo largo de los periodos analizados; por otra, a incorporar un objetivo de descripción de los juicios de los públicos sobre cada candidato, de cara a constatar los resultados que obtuvieran de la posible aplicación de este modelo. No obstante, su seguimiento es hipotético en esta fase dado que, aunque las organizaciones manifiesten que prefieren un modelo bidireccional de comunicación, en la práctica no siempre hay acuerdo sobre la calidad de la relación, generando incertidumbre (Walden et al., 2015, p. 532). 
Como no podía ser de otra manera hoy en día, este diálogo, entendimiento y acuerdo activos con los públicos puede fomentarse especialmente a través de las redes sociales. Este público, pionero en la adopción de redes sociales, desarrolla su aprendizaje político en nuevas fuentes de noticias, como Facebook, cuyas dinámicas de difusión e impacto en el conocimiento político de los jóvenes ya están logrando una considerable independencia de las de los medios tradicionales (Edgerly, Thorson y Wells, 2018, pp. 1055-1056). Paralelamente, en la llamada "política de la celebridad", las emociones y los sentimientos se convierten en fuente de afinidad, identificación y evaluación de los políticos (Loaden, Bromen y Xenos, 2016, p. 404). Este fenómeno interactúa con una nueva realidad, en la que el público joven alberga una nueva forma de entender la política, centrada más en los problemas concretos según éstos surgen, que en su sistematización y explicación a través del sentido que tradicionalmente proporcionaba la ideología (Manning, 2013). Algunas conclusiones invitan a formular un objetivo expresamente orientado a indagar en los procesos por los que el público joven realiza atribuciones sobre los candidatos electorales:

Está claro por nuestros hallazgos [...] que es probable que la efectividad del uso de redes

sociales en la movilización de apoyos, dependa de la receptividad de los políticos y celebridades políticas a las expectativas y exigencias de actuación de los ciudadanos jóvenes que se conectan en red. La mayor parte de la literatura existente se centra en el estudio de los políticos y famosos cuando usan los medios, en vez de en los ciudadanos jóvenes como agentes sociales activos en dar forma a la comunicación política y las prácticas de los representantes políticos. (Loaden et al., 2016, p. 414)

En el presente trabajo, se ha tratado de analizar cómo las variables del modelo de coorientación interactuaron con los marcos de percepción de los públicos ante las elecciones generales celebradas en los años 2015 y 2016 en España; cómo dichos marcos contextualizaron los debates electorales celebrados durante aquellas campañas, repercutiendo sobre la credibilidad percibida de cada candidato; y qué dimensiones trabajaron éstos últimos para lograr ser percibidos como creíbles.

\subsection{El enmarcado de los mensajes electorales}

Se denominan como frames (o marcos, en su traducción al español) a las estructuras básicas de entendimiento con las que dotamos de sentido a los acontecimientos; una estructura que permite además entender qué es lo relevante de cada situación o tema, proporcionándoles significado (Mueller, 2018, p. 25). Gracias a los frames o marcos, los individuos perciben, identifican y etiquetan las situaciones de interacción de su día a día. Esta propuesta tiene una gran acogida en los estudios sobre comunicación, porque los frames o marcos ayudan a comprender cómo influyen o cómo acepta la audiencia aquellas interpretaciones sugeridas por los medios sobre los acontecimientos públicos.

En esta línea, Robert Entman trató de sintetizar el recorrido de la teoría del framing en comunicación, definiendo el proceso de enmarcar o encuadrar como: "seleccionar algunos aspectos de la realidad percibida y hacerlos más prominentes en un texto comunicativo, de manera que promuevan definiciones particulares de los problemas, interpretaciones causales, evaluaciones morales y/o recomendaciones para el tratamiento del asunto descrito" (Entman, 1993, p. 52). El 
ciudadano vive en un ambiente comunicativo donde se elaboran los significados, se construyen las opiniones y se expresan las elecciones políticas, apunta Mazzoleni (2010, p. 232), quien añade que lo hace a través de tres fuentes de comunicación que son al mismo tiempo fuentes de influencia: los medios, la interacción social y los sujetos políticos. En lo que respecta a la primera de ellas, los medios llevan a cabo un enmarcado selectivo con el que pueden tornar las noticias con carga política en positivas, negativas, críticas o de apoyo (Watanabe, 2017, p. 225).

Pero no sólo los medios recurren al enmarcado. Los propios candidatos políticos pueden construir y afinar los frames con los que enmarcan sus mensajes, en un esfuerzo por conseguir que los debates políticos se produzcan en los términos que el candidato propone (Harris, 2010, p. 42). Lo que es más importante, Druckman (2001, p. 1061) establece que la credibilidad de la fuente de un mensaje es determinante a la hora de que los receptores asuman el frame que ésta pretende establecer.

\subsection{El estudio de la credibilidad percibida}

En este punto entra en escena la credibilidad de la fuente como factor determinante de la imagen de los candidatos y de los partidos políticos. Desde la perspectiva de Daniel Kahneman (2012, p. 35) hay dos formas de pensamiento que mediatizan nuestro comportamiento: el Sistema 1, que opera de manera rápida y automática, con poco o ningún esfuerzo y sin sensación de control voluntario; el Sistema 2, que centra la atención en las actividades mentales esforzadas que lo demandan, incluidos los cálculos complejos. Las operaciones del Sistema 2 están a menudo asociadas a la experiencia subjetiva de actuar, elegir y concentrarse. Ambos sistemas actúan como vasos comunicantes, donde el Sistema 2 tiene cierta capacidad para cambiar la manera de trabajar del Sistema 1 programando las funciones normalmente automáticas de la atención y la memoria (2012, pp. 37-38).

Robert Cialdini hace referencia a una reflexión de Daniel Kahneman acerca de que "nada en la vida es tan importante como crees que es mientras lo estás pensando" (Cialdini, 2017, p. 33). Este especialista continúa:

Las implicaciones de la afirmación de Kahneman se aplican muy bien a la práctica de la pre-

suasión, porque un comunicador que consigue que una audiencia se centre en un elemento

clave de un mensaje lo pre-carga con importancia. Esta forma de pre-suasión explica lo que

muchos ven como el papel principal (etiquetado como agenda setting) que los medios de

comunicación utilizan para influir en la opinión pública. El principio central de la teoría de la

agenda setting es que los medios rara vez producen cambios directamente; es mucho más

probable que persuadan indirectamente, al dar a temas y hechos seleccionados una mejor

cobertura que a otros temas y hechos. (Cialdini, 2017, pp. 33-34)

Los procesos de persuasión que influyan en los dos sistemas de pensamiento son de gran valor desde el punto de vista de la eficacia. Según la Escuela de Yale, el receptor realiza los juicios sobre la credibilidad de la fuente basándose en el conocimiento experto que le atribuye y cómo de fiable le parece (Hovland, Janis, y Kelley, 1953). Precisamente, la credibilidad ha sido descrita como "un activo crucial para los líderes políticos, y es clave para comprender el fracaso y el éxito en el competitivo mercado político" (Van Zuydam y Hendriks, 2018, p. 258). 
José Luis Arceo (1993) realizó una revisión de los mencionados estudios sobre la imagen de la fuente en comunicación política, delimitando tres factores: la credibilidad, el atractivo y el poder. Así, divide a su vez la credibilidad en tres subfactores: la competencia o conocimiento experto, la fiabilidad ética y la eficacia social (Arceo, 1993, p. 18). Puesto que estas dimensiones secundarias están en línea con las descritas en la literatura previa sobre credibilidad (Hovland y Weiss, 1951; Hovland et al., 1953), y a la luz de los comentarios de Van Zuydam y Hendriks (2018), se planteará el objetivo de realizar un seguimiento de las estrategias de credibilidad de cada candidato en las elecciones objeto de estudio. Dichas estrategias pretenden impactar en el contexto de percepción, dado que tratan de lograr unas atribuciones por parte de la audiencia, antes y después de los debates. Por su parte, Kim, Kiousis y Molleda (2015) constataron cómo los esfuerzos por dotar de atributos afectivos a los mensajes, impactaban en la credibilidad, la confianza y la autenticidad atribuidas a la fuente.

Sin embargo, ello no significa que se deban desdeñar los otros dos factores. El atractivo del candidato se basa en su notoriedad y, principalmente, en la semejanza que un miembro del público perciba con sus propias características sociodemográficas o estilo de vida. Todorov, Mandisodza, Goren y Hall (2005, p. 1623) afirman que se pueden predecir resultados electorales partiendo de las inferencias que evoque en los electores el aspecto facial de los candidatos. Este papel de la apariencia física se vuelve más importante cuando las elecciones se personalizan en los candidatos, "y cuando los costes de adquirir información sobre los candidatos son altos" (Lawson, Lenz, Baker y Myers, 2010, p. 562).

Respecto al factor del poder (Arceo, 1993, p. 18), incluye la capacidad decisoria del candidato, su estatus, apoyo sociopolítico y económico, e interés en la opinión del público.

\section{Objetivos}

La investigación parte de la hipótesis general de que los subfactores de la credibilidad, tal y como los describió José Luis Arceo (1993), son la variable determinante en la generación de los contextos de percepción de los debates electorales. Esta hipótesis se basa, igualmente, en la afirmación de la Escuela de Yale de que el componente básico de la credibilidad de la fuente es precisamente la aceptación del portavoz por parte del público (Hovland y Weiss, 1951). Para guiar hacia su validación o refutación, y en consonancia con el propósito y el problema definidos, se han establecido una serie de objetivos derivados. A lo largo de esta introducción se han ido exponiendo las contribuciones clave que han conducido hasta la formulación de los siguientes objetivos:

1. Observar la evolución de los citados contextos: Qué lectura hacían los medios y la ciudadanía.

2. Identificar cualquier variación en las estrategias de los candidatos para ganar credibilidad.

3. Detectar los parámetros por los que el público joven atribuye credibilidad a los candidatos.

4. Sintetizar los juicios realizados por el público sobre cada uno de los cuatro principales candidatos a las elecciones generales de 2015 y 2016.

\section{Metodología}

Para llevar a cabo la presente investigación, se ha empleado una metodología mixta, la cual conlleva la combinación de técnicas cuantitativas y cualitativas que pueden desplegarse simultáneamente, en un orden cronológico, o en un orden secuencial (Ongwuebuzie y Combs, 2011). En el caso del estudio que nos ocupa, se han combinado las técnicas cualitativas de los grupos de discusión y un análisis descriptivo de textos, con la técnica cuantitativa de los análisis de contenido. Su aplicación se ha llevado a cabo siguiendo el orden secuencial por el que se presentan en las próximas líneas. 
Mediante la técnica del grupo de discusión, se recogen los discursos de sus participantes a través de las interacciones y procesos de negociación entre ellos, con la intención última de obtener un marco general de información sobre las opiniones representativas, actitudes, sistemas simbólicos, etc. (Ballestín y Fàbregues, 2018, pp. 155-158). Respecto al análisis de contenido, es el método destinado al "análisis sistemático, objetivo y cuantitativo de las características del mensaje" (Neuendorf, 2017, p. 1). Esa sistematización que proporciona, sitúan al análisis de contenido como la técnica indicada para extraer datos objetivos del desempeño de los candidatos en redes sociales.

\subsection{Revisión de encuestas electorales y de opinión}

Como paso inicial, se procedió al análisis de las encuestas publicadas por las dos principales instituciones demoscópicas en España: El Centro de Investigaciones Sociológicas (CIS), como el organismo público que se dedica al estudio de la sociedad española; y la empresa Metroscopia (entidad que, durante el periodo analizado, trabajaba con el diario El País).

En ambos casos, se recogieron los estudios publicados en los dos meses previos a cada cita electoral, para obtener información relevante sobre: Intención de voto; principales problemas del país para los encuestados, y valoraciones de los cuatro candidatos. Los datos recabados contribuyen a arrojar más luz para interpretar la información obtenida del conjunto de técnicas de esta metodología mixta.

\subsection{Celebración de grupos de discusión}

Para comprender cómo opera el factor de credibilidad en las percepciones de la población española menor de 30 años, se organizaron dos grupos de discusión con actuantes de entre 19 y 29 años. Con la elección de esta técnica no se pretendió averiguar a posteriori las atribuciones de credibilidad que esta población realizara ante los debates estudiados, sino que, con intención exploratoria, los resultados se han empleado para una mejor comprensión del proceso de formación de impresiones sobre la credibilidad de los candidatos.

Se llevó a cabo un muestreo estratégico para conformar dos focus groups, dividiendo a los actuantes en un primer grupo con los más jóvenes, y con participantes procedentes de distintas regiones; y un segundo grupo formado exclusivamente por madrileños de 22 a 29 años de edad. Mediante este diseño, se pudo triangular cuáles eran los estados de opinión que se repetían en ambas sesiones y que, por lo tanto, tenían en común jóvenes con características bien diferenciadas.

En consecuencia, el primer grupo de discusión celebrado estuvo compuesto por jóvenes universitarios o recién egresados, residentes en la Comunidad de Madrid en el momento de su realización. En esta primera dinámica participaron cuatro mujeres de 22 años de edad (tres de ellas procedentes de Asturias, Córdoba y Murcia), una mujer de 19 años y un hombre de 22 (procedente de Extremadura). El muestreo estratégico para la segunda dinámica se realizó entre jóvenes titulados residentes dentro del área metropolitana de Madrid. Así, contó con la presencia de cuatro mujeres de 22 a 29 años de edad, un hombre de 27 años y otro de 29.

Ambos grupos arrancaron con la proyección de un fragmento del debate electoral del 13 de junio de 2016. En la secuencia, el periodista preguntaba a los cuatro candidatos qué harían para evitar una segunda repetición electoral. Una vez finalizado el fragmento, el dinamizador animaba a los actuantes a compartir sus percepciones, aplicando un guion basado en los subfactores de credibilidad expuestos. 


\subsection{Estudio descriptivo de las noticias de prensa}

Para ambos comicios, se analizaron los rasgos con los que la prensa escrita reflejó los respectivos contextos de percepción. Con este fin, se acudió a la hemeroteca para analizaron las noticias de portada sobre cada campaña, de tres cabeceras españolas de prensa (El País, El Mundo y ABC) durante dos periodos: Del 6 al 28 de diciembre de 2015, y del 13 de junio al 6 de julio de 2016. Los totales de piezas informativas analizadas pueden consultarse en la tabla 1.

Tabla 1. Piezas informativas de portada: Número total de unidades de análisis.

\begin{tabular}{|l|c|c|c|}
\hline & El Pais & El Mundo & $A B C$ \\
\hline $\begin{array}{l}\text { Periodo del 6 al 28 de } \\
\text { diciembre de 2015 }\end{array}$ & 186 & 207 & 132 \\
\hline $\begin{array}{l}\text { Periodo del 13 de junio } \\
\text { al 6 de julio de 2016 }\end{array}$ & 157 & 169 & 76 \\
\hline
\end{tabular}

Fuente: elaboración propia.

La inclusión en la muestra de unidades datadas en días posteriores a las elecciones permite tomar en consideración la posible persistencia de un contexto de percepción de la situación política que trascendiera a las respectivas campañas.

Se recurrió a un análisis descriptivo de la muestra (Berrocal, Redondo, Martín y Campos, 2014), con el que recoger sus principales rasgos de interés para el propósito y los objetivos de la investigación. Durante dicho análisis, se registró la posible presencia de ocho componentes que indicarían una clara tendencia hacia la desinformación, afectando por lo tanto a la credibilidad de la fuente o del mensaje:

1. Agresividad: cuando un candidato o partido atacaba a otros actores políticos, o se defendía de ataques externos.

2. Miedo: cuando el candidato o partido hablaran de una amenaza externa; o, al menos, cuando se hablase de cómo proteger a la gente de ella.

3. Alegría: los protagonistas de la noticia aparecen sonriendo, se hace referencia a la acción de sonreír o se manifiesta un juicio positivo.

4. Enfado: cuando el candidato, el partido o el propio medio formularan en la noticia una protesta de cualquier índole.

5. Mentira: referencias de un candidato o partido a afirmaciones falsas de los rivales; aparición de afirmaciones falsas en el discurso del candidato o en la cobertura dada por el periódico en la noticia.

6. Tristeza/decepción: referencias a eventos no deseados que ya hubieran tenido lugar. Los comentarios favorables a la idea de cambio también fueron codificados como decepción, dado que ilustran la insatisfacción del candidato, del partido o del propio medio con la situación de la que se hablara.

7. Populismo: comentarios del candidato o del medio que reforzaran la idea de pertenencia de la audiencia a una comunidad; ejemplos de ciudadanos específicos a los que recurriera el candidato.

\subsection{Análisis de contenido de cuentas de Twitter}

Para recoger cómo trabajaron los candidatos las atribuciones de credibilidad a través de redes sociales, se ha escogido Twitter ante la realidad señalada por Chaves-Montero y Gadea (2017, p. 13), 
de acuerdo con la cual los partidos y candidatos se decantan por conceder una mayor relevancia a esta plataforma frente a otras alternativas. Se han analizado los tuits y retuits lanzados desde los perfiles de los cuatro candidatos en dos periodos: Del 1 al 20 de diciembre de 2015, y del 30 de mayo al 19 de junio de 2016 (tabla 2). Estas fechas comprenden las dos semanas previas y la semana posterior a cada debate, para comparar resultados entre los periodos que les anteceden y los que les siguen.

Tabla 2. Corpus de tuits de cada candidato: Número total de unidades de análisis.

\begin{tabular}{|l|c|c|c|c|}
\hline & Mariano Rajoy & Pedro Sánchez & Pablo Iglesias & Albert Rivera \\
\hline $\begin{array}{l}\text { Periodo del 1 al 20 de } \\
\text { diciembre de 2015 }\end{array}$ & 880 & 722 & 257 & 528 \\
\hline $\begin{array}{l}\text { Periodo del 30 de mayo } \\
\text { al 19 de junio de 2016 }\end{array}$ & 774 & 754 & 195 & 774 \\
\hline
\end{tabular}

Fuente: Elaboración propia.

Se usaron dos plataformas para recuperar los tuits y disponer de ellos como unidades de análisis: El sistema Cecubo Metrics y TweetDeck. El primero de ellos es capaz de proporcionar un volcado de todos los mensajes de los perfiles que interesen al investigador, durante el periodo de tiempo que éste indique. En caso de que interesara para su propósito, los resultados de la búsqueda de Cecubo Metrics no sólo recogen los tuits de la cuenta en cuestión, sino todos aquellos en los que resultase mencionada. Sólo en el caso puntual de los mensajes de Albert Rivera entre los días 6 y 9 de diciembre de 2015, se cubrió la ausencia de resultados en Cecubo Metrics con los que arrojó la plataforma TweetDeck, una herramienta de gestión de Twitter compatible con el lenguaje de consultas que opera en esta red social.

Se desarrolló un libro de códigos con las siguientes definiciones para los factores de imagen:

1. Conocimiento experto: se codificó cuando el candidato demostraba dominio sobre un tema, o atacaba la falta de conocimiento de un adversario. Ejemplos: presentación de datos contrastados; desmentido de datos de un tercero; valoraciones sobre capacidades de gestión.

2. Fiabilidad ética: alusiones del candidato a su propia honestidad, o cuestionamiento de la de sus rivales. Ejemplos: alusión a compromisos propios o de sus adversarios; menciones a la corrupción propia o ajena; a la denuncia de conductas o intenciones, y a pedir la confianza.

3. Eficacia social: desarrollo de argumentaciones, formulación de propuestas, realización de acciones o invitaciones al público a realizarlas.

4. Atractivo: presencia del candidato o sus compañeros en un vídeo o fotografía; muestras de su estilo de vida o de su vida personal; imágenes del aforo en actos públicos; agradecimientos del candidato a terceros, y muestras de interés por sus opiniones.

5. Poder: en este caso, la presencia de una serie de términos bastaba para codificar la categoría: "Gobierno", uso del verbo "poder" en relación con acciones del partido o el candidato, administración de premios o castigos, aparición de mandatarios internacionales...

Cada una de las categorías fue codificada en cada tuit en función de qué elementos la denotaban: si se observaba en la imagen, en el texto, en su primera frase, en todos ellos... Con estos criterios, se compuso la siguiente escala del 0 al 4:

1. Ausencia de la categoría en el tuit.

2. Categoría presente en frases secundarias del texto del tuit, en la entradilla del enlace a una web, o presencia testimonial en la imagen o vídeo que se incluyeron. 
3. Presencia de la categoría por las menciones a perfiles de terceros, así como en hashtags introducidos a mitad o al final del mensaje. La categoría también puede aparecer en el contenido al que enlaza el link incluido en el tuit; ocupando hasta un $30 \%$ del vídeo incorporado; o, por último, sin ocupar más de la quinta parte de la imagen subida por el candidato.

4. La categoría abre el tuit en su primera frase, pero sin protagonizar cualquier posible imagen, vídeo, enlace o elemento incrustado. También se codifica con esta intensidad el caso opuesto: Que el ítem protagonice la imagen, vídeo, enlace o incrustado, pero no aparezca en la frase inicial del mensaje.

5. La categoría se registra en todos los componentes del tuit. Esta puntuación máxima también se registra si la categoría se observa en la frase de apertura, sin que el tuit contenga ningún elemento multimedia adicional.

\section{Resultados}

Entre finales de 2015 y junio de 2016, las encuestas recogieron cómo el contexto de percepción empeoraba sensiblemente en tres aspectos clave: la situación económica, la preocupación por la corrupción y la valoración de los líderes políticos. A lo largo de todo ese tiempo, los componentes de miedo y agresividad son una constante en los diarios analizados, al igual que una serie de patrones en los mensajes que lanzaba cada candidato a través de Twitter. Los comentarios vertidos en los grupos de discusión por votantes jóvenes añaden a este escenario posturas muy críticas con los líderes políticos y sus partidos; y, en especial, con las formaciones tradicionales. Sin embargo, estas últimas opiniones no siempre se trasladaron a las evaluaciones sobre la credibilidad de cada candidato.

\subsection{Información relevante de las encuestas}

Según las series del CIS, en octubre de 2015 los tres principales problemas para los españoles eran, por este orden: El paro, la corrupción y la situación económica. Sin embargo, la corrupción era el problema que los ciudadanos preveían más determinante electoralmente. En noviembre, esta preocupación llegó a su punto más alto, con un 40,1\% de menciones cuando los encuestados eran preguntados por sus inquietudes. Dicha evolución coincidió con un acercamiento en intención de voto de los nuevos partidos, Podemos y Ciudadanos (que se colocó en segunda posición), a PP y PSOE.

En el sondeo de diciembre, cuando la preocupación por la corrupción volvió a descender ligeramente (quedándose en el 38,8 \%), los dos partidos tradicionales se distanciaron de nuevo. Esta correspondencia se da especialmente con Ciudadanos, ya que Unidos Podemos vio cómo aumentaba su intención de voto de mayo a junio de 2016 (cuando las preocupaciones seguían siendo las mismas), a pesar de que la preocupación por la corrupción descendía (de un 47,8 \% a un 44,9\%, pero siempre cifras superiores a las de finales de 2015). Respecto a la situación económica, el incremento de las percepciones negativas en el CIS preelectoral de junio de 2016 frente al de diciembre de 2015, coincide con el de la preferencia de los encuestados por Unidos Podemos sobre Ciudadanos.

Entre diciembre de 2015 y junio de 2016 cae la valoración de los cuatro líderes. En ambos sondeos del CIS, Rivera es el candidato mejor valorado, con un 4,98 y un 4,17, respectivamente. Todos conservaron la posición con un descenso en la nota media. El sondeo de Metroscopia del 1 de noviembre de 2015 también señalaba a Rivera como el preferido por los españoles, pero le seguían Rajoy, Sánchez e Iglesias, por este orden. Para la encuesta de Metroscopia del 7 de junio de 2016, la situación había variado notablemente: Sánchez era el favorito, seguido de Rajoy, Rivera e Iglesias. 
Respecto a las previsiones de los encuestados para las elecciones, según el estudio preelectoral del CIS de octubre-noviembre de 2015, el 52,4\% pensaba que el 20-D ganaría el PP. La victoria de los populares parecía todavía más clara para el 26-J, según el preelectoral de mayo de 2016: Un 67,4\% de los encuestados la vaticinaba.

\subsection{Tratamiento de las campañas en prensa}

Tomando como referencia el registro de los ocho componentes de desinformación descritos en la metodología, se puede afirmar que los tres diarios seleccionados reprodujeron marcos con el potencial de minar la credibilidad de los candidatos o sus mensajes. Esta dinámica fue especialmente acentuada en los casos de El País y El Mundo. En conjunto entre las dos campañas, éste último diario también se sitúa en cabeza en cuanto a la presencia del componente populista, mientras que $A B C$ es el diario que menos lo plasma.

En el caso de El País, los componentes de enfado y miedo se detectaron con llamativa frecuencia durante la campaña de diciembre, sin apenas transmisión alguna del componente de alegría, en los términos en los que fue definido para la aplicación de esta técnica (tabla 3). No obstante, El País es el periódico en el que se ha recogido un nivel más bajo de agresividad, en comparación con las otras dos cabeceras. Para las elecciones de junio de 2016, los componentes de mentira y agresividad fueron los más registrados, dejando las categorías de miedo y enfado ligeramente por detrás.

Tabla 3. Número de veces que las categorías desinformativas fueron registradas en noticias del diario El País.

\begin{tabular}{|l|c|c|c|}
\hline & $\begin{array}{c}\text { Campaña de } \\
\text { diciembre de } 2015\end{array}$ & $\begin{array}{c}\text { Campaña de junio } \\
\text { de } 2016\end{array}$ & Totales \\
\hline Agresividad & $(17,74 \%) 33\left(4^{\circ}\right)$ & $(22,29 \%) 35\left(2^{\circ}\right)$ & $(19,83 \%) 68\left(3^{\circ}\right)$ \\
\hline Miedo & $(22,58 \%) 42\left(2^{\circ}\right)$ & $(17,83 \%) 28\left(3^{\circ}\right)$ & $(20,41 \%) 70\left(2^{\circ}\right)$ \\
\hline Alegría & $(1,61 \%) 3$ & $(3,82 \%) 6$ & $(2,62 \%) 9$ \\
\hline Enfado & $(23,66 \%) 44\left(1^{\circ}\right)$ & $(14,01 \%) 22\left(4^{\circ}\right)$ & $(19,24 \%) 66\left(4^{\circ}\right)$ \\
\hline Mentira & $(20,97 \%) 39\left(3^{\circ}\right)$ & $(29,94 \%) 47\left(1^{\circ}\right)$ & $(25,07 \%) 86\left(1^{\circ}\right)$ \\
\hline Tristeza/decepción & $(5,38 \%) 10$ & $(3,82 \%) 6$ & $(4,67 \%) 16$ \\
\hline Populismo & $(8,06 \%) 15$ & $(8,28 \%) 13$ & $(8,16 \%) 28$ \\
\hline Total & 186 artículos & 157 artículos & 343 artículos \\
\hline
\end{tabular}

Fuente: elaboración propia.

Los resultados para El Mundo muestran de nuevo el predominio de componentes emocionales negativos en la información electoral. En este caso, la agresividad sí que es el componente predominante, si bien la presencia de la categoría del miedo se atenuó entre diciembre de 2015 y junio de 2016 (tabla 4).

Tabla 4. Número de veces que las categorías desinformativas fueron registradas en noticias del diario El Mundo.

\begin{tabular}{|l|c|c|c|}
\hline & $\begin{array}{c}\text { Campaña de } \\
\text { diciembre de } 2015\end{array}$ & $\begin{array}{c}\text { Campaña de junio } \\
\text { de } 2016\end{array}$ & Totales \\
\hline Agresividad & $(25,12 \%) 52\left(1^{\circ}\right)$ & $(23,08 \%) 39\left(1^{\circ}\right)$ & $(24,20 \%) 91\left(1^{\circ}\right)$ \\
\hline Miedo & $(23,19 \%) 48\left(2^{\circ}\right)$ & $(15,38 \%) 26\left(4^{\circ}\right)$ & $(19,68 \%) 74\left(3^{\circ}\right)$ \\
\hline Alegría & $(3,86 \%) 8$ & $(11,24 \%) 19$ & $(7,18 \%) 27$ \\
\hline Enfado & $(14,98 \%) 31\left(4^{\circ}\right)$ & $(17,16 \%) 29\left(3^{\circ}\right)$ & $(15,96 \%) 60\left(4^{\circ}\right)$ \\
\hline Mentira & $(19,32 \%) 40\left(3^{\circ}\right)$ & $(20,71 \%) 35\left(2^{\circ}\right)$ & $(19,95 \%) 75\left(2^{\circ}\right)$ \\
\hline
\end{tabular}


RLCS, Revista Latina de Comunicación Social, 76, 17-37

[Investigación] DOI: 10.4185/RLCS-2020-1435 | ISSN 1138-5820 | Año 2020

\begin{tabular}{|l|c|c|c|}
\hline Tristeza/decepción & $(2,42 \%) 5$ & $(5,33 \%) 9$ & $(3,72 \%) 14$ \\
\hline Populismo & $(11,11) 23$ & $(7,10 \%) 12$ & $(9,31 \%) 35$ \\
\hline Total & 207 artículos & 169 artículos & 376 \\
\hline
\end{tabular}

Fuente: elaboración propia.

Respecto al diario $A B C$, la muestra se compuso principalmente de noticias de diciembre de 2015. Las portadas de $A B C$ cubrieron ambas campañas con un notable predominio de los componentes de agresividad y miedo (tabla 5).

Tabla 5. Número de veces que las categorías desinformativas fueron registradas en noticias del diario $A B C$.

\begin{tabular}{|l|c|c|c|}
\hline & $\begin{array}{c}\text { Campaña de } \\
\text { diciembre de } 2015\end{array}$ & $\begin{array}{c}\text { Campaña de junio } \\
\text { de 2016 }\end{array}$ & Totales \\
\hline Agresividad & $(29,54 \%) 39\left(1^{\circ}\right)$ & $(32,89 \%) 25\left(1^{\circ}\right)$ & $(30,77 \%) 64\left(1^{\circ}\right)$ \\
\hline Miedo & $(21,21 \%) 28\left(2^{\circ}\right)$ & $(17,11 \%) 13\left(2^{\circ}\right)$ & $(19,71 \%) 41\left(2^{\circ}\right)$ \\
\hline Alegría & $(6,82 \%) 9$ & $(3,95 \%) 3$ & $(5,77 \%) 12$ \\
\hline Enfado & $(13,64 \%) 18\left(4^{\circ}\right)$ & $(14,47 \%) 11\left(4^{\circ}\right)$ & $(13,94 \%) 29\left(4^{\circ}\right)$ \\
\hline Mentira & $(14,39 \%) 19\left(3^{\circ}\right)$ & $(15,79 \%) 12\left(3^{\circ}\right)$ & $(14,90 \%) 31\left(3^{\circ}\right)$ \\
\hline Tristeza/decepción & $(6,82 \%) 9$ & $(2,63 \%) 2$ & $(5,29 \%) 11$ \\
\hline Populismo & $(7,58 \%) 10$ & $(13,16 \%) 10$ & $(9,62 \%) 20$ \\
\hline Total & 132 artículos & 76 artículos & 208 artículos \\
\hline
\end{tabular}

Fuente: elaboración propia.

\subsection{Síntesis de los grupos de discusión}

En retrospectiva, los jóvenes actuantes recordaron cómo la llegada de dos nuevos partidos elevó considerablemente su interés por los debates (en especial, por el del 14 de junio de 2016, el primero con los cuatro candidatos a la presidencia). El desgaste de la credibilidad de PP y PSOE estuvo muy extendido en ambos grupos, pero la falta de eficacia social percibida fue común para todos los candidatos: los participantes consideraron que los líderes políticos miraban ante todo por sus propios intereses. La fiabilidad de las declaraciones de los actuantes sobre estas cuestiones, al igual que sobre las atribuciones de credibilidad a los líderes políticos, se refuerza gracias a que fueron recogidas inmediatamente a continuación de la proyección del fragmento inicial del debate de junio de 2016.

En cuanto a los hallazgos sobre cómo era percibido cada candidato, Mariano Rajoy se revela como una persona natural y sincera para la mayoría; pero también como un político falto de carisma y liderazgo. Rajoy encabezaría el reconocimiento de conocimiento experto y competencia, gracias a la experiencia de gobierno ya acumulada. Cuando en un grupo surgían los problemas de corrupción del PP, intervenían participantes que atribuían la falta de fiabilidad ética principalmente al partido, y no tanto al candidato.

Los casos de Pedro Sánchez y Albert Rivera son similares, en cuanto a que ambos comparten un importante punto débil a ojos de los actuantes jóvenes: un exceso de artificialidad que les conduce a perder atractivo y parecer altivos o prepotentes. Aunque no existió consenso a la hora de reconocerlo como un candidato creíble, la actuación de Rivera en el fragmento proyectado fue la menos comentada, con bajos niveles de recuerdo. Es percibido como un líder bien formado, pero inexperto en la gestión pública. 
Curiosamente, aunque los actuantes detectaban en Pablo Iglesias una clara preparación previa para presentarse como un político creíble, este hecho no jugó en contra de las percepciones de credibilidad, al contrario que en los casos de Rivera y Sánchez. Los participantes tendieron a reconocerle una gran capacidad de expresión oral, pero escaso conocimiento experto para gobernar.

Aunque la mayoría de los actuantes rechazó que los debates influyeran en su intención de voto, los participantes mostraron una gran diversidad en este aspecto. En la segunda dinámica, algunos reconocieron qué utilidad observaban en este formato: descartar candidatos en vez de decidirse por uno, al poder observarlos en un entorno menos controlado que otras acciones de campaña.

\subsection{Análisis de los perfiles en Twitter de los candidatos}

Gracias a la definición precisa de los ítems y sus grados de intensidad en el libro de códigos, se han podido obtener una serie de hallazgos singulares que, a continuación, se procede a destacar.

En Twitter, el subfactor de eficacia social fue el más recurrente para los cuatro candidatos, con una drástica ventaja frente al resto de variables. Todos destinaron la mayor parte de sus tuits a difundir propuestas programáticas, o bien a mostrar dinamismo mediante sus propias actividades (o mediante ataques a un adversario y respuestas a los de éste). Todas ellas fueron acciones contempladas en el libro de códigos para consignar la categoría "Eficacia Social".

Sin embargo, a partir de este patrón común, debemos destacar algunas especificidades para cada candidato. Mariano Rajoy empleó abiertamente su perfil de Twitter para reforzarse como un líder con conocimiento experto. Además, esta categoría del libro de códigos obtuvo registros más elevados de media en aquellos tuits con referencia a un debate electoral. Obsérvense estas apreciaciones en los resultados del periodo previo al debate a cuatro de junio de 2016 (tabla 6).

Tabla 6. Clasificación por el grado de intensidad (del 0 al 4) de la categoría "Conocimiento Experto/Competencia", en tuits con presencia de la categoría "Debates" (periodo del 30 de mayo al 13 de junio de 2016).

\begin{tabular}{|l|c|c|c|}
\hline & $\begin{array}{c}\text { Conocimiento } \\
\text { experto/ } \\
\text { competencia }\end{array}$ & Fiabilidad ética & $\begin{array}{c}\text { Dinamismo/eficacia } \\
\text { social }\end{array}$ \\
\hline 1. Mariano Rajoy & 1.68 & 0.36 & 3.34 \\
\hline 2. Pedro Sánchez & 0.93 & 0 & 3.07 \\
\hline 3. Pablo Iglesias & 0 & 0 & 3.14 \\
\hline 4. Albert Rivera & 0.23 & 1 & 3.05 \\
\hline
\end{tabular}

Fuente: elaboración propia.

Una tendencia igualmente singular es la que se da con el factor del atractivo en los tuits de Pablo Iglesias. Se trata sin lugar a dudas del candidato que más recurre a él en redes sociales, con independencia de que la eficacia social sigue siendo la categoría de análisis con puntuación media más alta. En especial, los registros de la categoría "Atractivo" son sensiblemente superiores a los de cualquier otro candidato tras el debate del 14 de junio de 2016 (tabla 7). 
RLCS, Revista Latina de Comunicación Social, 76, 17-37

[Investigación] DOI: 10.4185/RLCS-2020-1435 | ISSN 1138-5820 | Año 2020

Tabla 7. Clasificación por el grado de intensidad (del 0 al 4) de la categoría "Atractivo" (periodo del 14 al 20 de junio de 2016).

\begin{tabular}{|l|c|c|c|c|c|}
\hline & Atractivo & $\begin{array}{c}\text { Conocimiento } \\
\text { experto/ } \\
\text { competencia }\end{array}$ & $\begin{array}{c}\text { Fiabilidad } \\
\text { ética }\end{array}$ & $\begin{array}{c}\text { Dinamismo/ } \\
\text { eficacia social }\end{array}$ & $\begin{array}{c}\mathrm{N}^{\mathrm{o}} \text { de mensajes } \\
\text { analizados }\end{array}$ \\
\hline 1. Mariano Rajoy & 3.19 & 0.31 & 0.25 & 2.33 & 50 \\
\hline 2. Pedro Sánchez & 2.38 & 0.12 & 0.77 & 2.88 & 345 \\
\hline 3. Pablo Iglesias & 2.28 & 0.55 & 0.11 & 3.06 & 367 \\
\hline 4. Albert Rivera & 2.23 & 0.34 & 0.55 & 3.13 & 300 \\
\hline
\end{tabular}

Fuente: elaboración propia.

Por último, merece la pena repasar el recurso de cada candidato al subfactor de fiabilidad ética. Los registros de Mariano Rajoy en dicha categoría son casi testimoniales: entre 0,1 y 0,2 puntos sobre 4 para cualquiera de los periodos analizados. Fueron Pablo Iglesias y Pedro Sánchez quienes lideraron su uso durante la campaña para las elecciones del 20 de diciembre de 2015. Por su parte, Albert Rivera les relevó en la campaña de junio de 2016, convirtiéndose en el candidato que más aludía a la honestidad y sinceridad propias o ajenas.

Tal y como sucedía con el conocimiento experto de Rajoy, Sánchez alude todavía más a cuestiones de fiabilidad ética en aquellos tuits relacionados con los debates. En esta línea, la categoría de fiabilidad ética se acerca al empate con la de eficacia social, en aquellos mensajes publicados tras el cara a cara con Rajoy (tabla 8).

Tabla 8. Clasificación por el grado de intensidad (del 0 al 4) de la categoría "Fiabilidad Ética", en tuits con presencia de la categoría "Debates" (periodo del 15 al 20 de diciembre de 2015).

\begin{tabular}{|l|c|c|c|}
\hline & $\begin{array}{c}\text { Conocimiento } \\
\text { experto/ } \\
\text { competencia }\end{array}$ & Fiabilidad ética & $\begin{array}{c}\text { Dinamismo/eficacia } \\
\text { social }\end{array}$ \\
\hline 1. Mariano Rajoy & 2.23 & 0 & 3 \\
\hline 2. Pedro Sánchez & 0 & 0.6 & 3.6 \\
\hline 3. Pablo Iglesias & 0 & 1 & 4 \\
\hline 4. Albert Rivera & 0 & 0.32 & 3.5 \\
\hline
\end{tabular}

Fuente: elaboración propia.

\section{Discusión}

En su análisis de la campaña de 2015, López-García (2016, p. 163) concluía que los candidatos usaron la red social Twitter principalmente para cohesionar a sus seguidores y simpatizantes, y para promocionar las apariciones del candidato en medios y mítines. Son precisamente este tipo de contenidos los que dispararon el registro de la categoría "Eficacia Social", de acuerdo a su definición en el libro de códigos. Para generar el contexto de percepción adecuado, los candidatos tienen que haber trabajado la credibilidad percibida con acierto, previamente a los debates electorales. La descompensación entre el subfactor de eficacia social y el resto de variables analizadas evidencia que ese no fue el caso en las campañas de 2015 y 2016, evidenciando su fracaso generalizado a la hora de dotarse del necesario factor de credibilidad. Además, para cualquier equipo de campaña, la discrepancia entre los esfuerzos volcados en revestirse de eficacia social, y su nulo reconocimiento por los votantes jóvenes, debería ser el fenómeno más preocupante de cuantos se han hallado. 
El primer objetivo de la investigación pasaba por describir la evolución del contexto de percepción en el que se desarrollaron los debates electorales de 2015 y 2016. En ambos casos, los medios de comunicación sólo contribuyeron con componentes negativos susceptibles de fomentar la desinformación: Las grandes cabeceras reforzaron una serie de sentimientos capaces de actuar como elementos desinformadores. Hubo una agresividad generalizada en los textos publicados durante ambas campañas, si bien cabe destacar la disminución de elementos susceptibles de generar miedo en los lectores ante la incertidumbre política. En general, el papel de la prensa en la construcción del marco de percepción tampoco ayudó a un modelo de relación de doble flujo. La prensa contribuyó a generar un clima de crispación y desconfianza en ambas campañas, minando las probabilidades de atribuir credibilidad a los candidatos.

Esta revisión de las portadas de la prensa se encuentra en la misma línea que la conclusión de García Marín (2015, pp. 155-156), según la cual los medios españoles no reflejaron fidedignamente los debates electorales. En el caso de la televisión, las cadenas tendían a señalar los momentos conflictivos de los debates en el caso de la televisión; en cambio, la prensa se decantaba por un lenguaje evocador de la competitividad. Este último comentario pone sobre la misma senda que los elevados registros del componente de agresividad.

En lo referente a la evolución del contexto de percepción, merece la pena señalar cómo, en los grupos de discusión, los jóvenes tendieron a manifestar el hastío que les provocó una segunda campaña electoral en apenas seis meses. Las encuestas revelaron que el paro, la corrupción y la mala situación económica eran los problemas que más preocupaban a los españoles durante los meses previos a ambas citas electorales. Sin embargo, ningún candidato era lo bastante creíble para afrontarlos, ya que ninguno aprobaba cuando se pedía a los encuestados que les pusieran nota. Aunque los votantes contaban con la victoria del Partido Popular, se observa una correlación entre la preocupación por la corrupción y el voto a los nuevos partidos, en especial a Ciudadanos. Del mismo modo, la percepción de empeoramiento de la situación económica pareció beneficiar a Podemos.

Por lo que respecta al segundo objetivo, ningún candidato modificó sustancialmente su estrategia de credibilidad ante lo sucedido en cada debate electoral, con la notable excepción de Pedro Sánchez en diciembre de 2015: su giro hacia el subfactor de la fiabilidad ética (en alta correlación con la aparición del PP en sus tuits) se produjo inmediatamente después del cara a cara con Rajoy. En el futuro, merecerá la pena investigar el peso específico de la fiabilidad ética en el conjunto del factor de credibilidad. Para ello, se podrán tomar como referencia las campañas electorales más recientes.

En líneas generales, el público joven se interesó por los debates tomados como referencia en esta investigación. Ahora bien, toda influencia sobre su decisión de voto se redujo al descarte de candidatos que no les resultaran creíbles. Pero el resultado más llamativo es el rechazo a aquellos candidatos que parecían ejercer mayor control sobre su comunicación verbal o no verbal, para causar una buena impresión. Por el contrario, los actuantes jóvenes tendieron a valorar positivamente la naturalidad de Mariano Rajoy. Un estudio de las primarias a las elecciones presidenciales estadounidenses de 2008 ya señalaba que la percepción de manipulación o engaño de manos del candidato, correlacionaba negativamente con las percepciones de competencia, buena voluntad y confianza (Teven, 2008, p. 392). Es decir, la comunicación de cualidades positivas es lo que marca la diferencia, y no los intentos del candidato por aparentar las que no posee. En próximas investigaciones, se averiguará si estos patrones se reproducen en públicos de otros segmentos de edad.

El cuarto y último objetivo de la investigación pasaba por obtener una síntesis de los juicios del público sobre cada candidato. La naturalidad de Mariano Rajoy ante la cámara consigue inspirar 
confianza y honestidad entre el público joven. Globalmente, la credibilidad atribuida a Albert Rivera tendió a ser poca, si bien existen opiniones encontradas entre el público. Tanto Rivera como Pedro Sánchez sufrieron una falta de credibilidad por la artificialidad que transmitían. Curiosamente, a pesar de que se reconoce que Pablo Iglesias también llega a los debates con un gran trabajo de preparación, él sí resultó creíble para buena parte del público joven en el periodo analizado. En gran medida, los jóvenes le reconocen sinceridad y fiabilidad ética. En la actualidad, sería posible indagar acerca de la evolución de la credibilidad atribuida a cada uno de estos líderes, desde las elecciones analizadas en el presente artículo hasta hoy. La academia y la profesión podrían aprender mucho de sus respectivos aciertos y fallos en sus esfuerzos de relaciones públicas políticas.

\section{Conclusiones}

La presente investigación se iniciaba con objeto de analizar el contexto de percepción de unos debates electorales singulares por celebrarse en un nuevo tablero político, al incorporarse dos nuevos partidos con potencial movilizador del voto; y, en el caso del de junio de 2016, tras una situación de bloqueo nunca antes vivida en España para la formación de gobierno. Todo ello en pleno auge de las redes sociales como canal para la comunicación política. De los resultados expuestos se concluye que los candidatos habrían sacado más partido de una aproximación completa a sus diferentes subfactores, en vez de volcarse infructuosamente en la eficacia social. Para los candidatos, no resultó efectivo basar todos sus esfuerzos en la eficacia social y trabajarla mediante un exceso de artificialidad. En consecuencia, el marco de percepción de ambos debates no fue propicio para lograr que el público les atribuyera credibilidad ante su desempeño en ellos.

Las redes sociales sirvieron de plataforma para los esfuerzos de los candidatos por revestirse de eficacia social. Llamativamente, los jóvenes denuncian principalmente cómo los líderes políticos carecían de este subfactor. Ello implicaría unos grados muy pobres de entendimiento mutuo y acuerdo entre candidatos y electores. De modo similar operan los recelos hacia quienes más controlaron su credibilidad proyectada. Por otra parte, los resultados reflejan cómo Mariano Rajoy generó con éxito a través de Twitter un marco de percepción positivo acerca de su conocimiento experto.

Por último, de haberse mostrado más cercanos, los candidatos podrían haber atenuado el clima de sentimientos negativos que reflejaron los principales diarios durante las campañas. En consecuencia, conviene que los candidatos eviten encorsetamientos para mostrarse ante los votantes con más naturalidad.

\section{Referencias}

Aaldering, L.; Van der Meer, T. \& Van der Brug, W. (2018). Mediated leader effects: The impact of newspapers' portrayal of party leadership on electoral support. The International Journal of Press/Politics, 23(1), 70-94. https://www.doi.org/10.1177/1940161217740696

Arceo Vacas, J. L. (Dir.) (1993). Campañas electorales y publicidad política en España (19761991). Barcelona: ESRP-PPU.

Ballesteros Herencia, C. A. (2017, julio). Movilización en las campañas electorales a través de las redes sociales. Las elecciones generales españolas de 2015. Ponencia presentada en la $15^{\mathrm{a}}$ conferencia de la International Association for Media and Communication Research, Cartagena de Indias, Colombia. http://uvadoc.uva.es/handle/10324/26049 
Berrocal, S.; Redondo, M.; Martín, V. y E. Campos (2014). La presencia del infoentretenimiento en los canales generalistas de la TDT española. Revista Latina de Comunicación Social, (69), 85103. https://www.doi.org/10.4185/RLCS-2014-1002

Broom, G. M. (1977). Coorientational measurement of public relations. Public Relations Review, 3(4), 110-119. https://www.doi.org/10.1016/S0363-8111(77)80010-6

Cialdini, R. (2017). Pre-Suasion. A revolucionary way to influence and persuade. Simon \& Schuster.

Chaves-Montero, A. y Gadea, W. F. (2017). Uso, efectividad y alcance de la comunicación política en las redes sociales. En: A. Chaves-Montero (Ed.), Comunicación política y redes sociales (pp. 13-32). Egregius.

Coleman, S. \& Moss, G. (2016). Rethinking election debates: What citizens are entitled to expect. The International Journal of Press/Politics, 21(1), 3-24.

https://www.doi.org/10.1177/1940161215609732

Druckman. J. N. (2001). On the limits of framing effects: who can frame? The Journal of Politics, 63(4), 1041-1066. https://www.doi.org/10.1111/0022-3816.00100

Edgerly, S. L.; Thorson, K. \& Wells, C. (2018). Young citizens, social media, and the dynamics of political learning in the U.S. presidential primary election. American Behavioral Scientist, 62(8), 1042-1060. https://www.doi.org/10.1177/0002764218764236

Entman, R. M. (1993). Framing: Toward clarification of a fractured paradigm. Journal of Communication, 43(4), 51-58. https://www.doi.org/10.1111/j.1460-2466.1993.tb01304.x

Eveland, Jr., W. P. \& Hutchens, M. J. (2013). The role of conversation in developing accurate political perceptions: A multilevel social network approach. Human Communication Research, 39(4), 422-444. https://www.doi.org/10.1111/hcre.12011

Harris, D. B. (2010). Partisan framing in legislative debates. En: B. F. Schaffner y P. J. Sellers (Eds.): Winning with words: The origins and impact of political framing. Routledge.

Kahneman, D. (2012). Pensar rápido, pensar despacio. Penguin Random House.

Kim, J. Y.; Kiousis, S. \& Molleda, J. C. (2015). Use of affect in blog communication: Trust, credibility, and authenticity. Public Relations Review, 41(4), 504-507. https://www.doi.org/10.1111/hcre.12011

Lawson, J.; Chappell, H.; Lenz, G. S.; Baker, A. \& Myers, M. (2010). Looking like a winner: Candidate appearance and electoral success in new democracies. World Politics, 62(4), 561-593. https://www.doi.org/10.1017/S0043887110000195

Loader, B.; Vromen, A. \& Xenos, M. (2014). The networked young citizen: Social media, political participation and civic engagement. Information Communication and Society, 17(2), 143-150. https://www.doi.org/10.1080/1369118X.2013.871571 
López-García, G.; Llorca-Abad, G.; Valera-Ordaz, L. y Peris-Blanes, À. (2018). Los debates electorales, ¿el último reducto frente la mediatización? Un estudio de caso de las elecciones generales españolas de 2015. Palabra Clave, 21(3), 772-797.

https://www.doi.org/10.5294/pacla.2018.21.3.6

Manning, N. (2013). The relational self and the political engagements of young adults. Journal of Sociology, 50(4), 486-500. https://www.doi.org/10.1177/1440783312467094

Mazzoleni, G. (2010). La comunicación política. Alianza Editorial.

McLeod, J. M. \& Chafee, S. H. (1973). Interpersonal approaches to communication research. American Behavioral Scientist, 16(4), 469-500. https://www.doi.org/10.1177/000276427301600402

Mueller, F. (2018). Taking Goffman seriously: Developing strategy-as-practice. Critical Perspectives on Accounting, (53), 16-30. https://www.doi.org/10.1016/j.cpa.2017.03.009

Ongwuebuzie, A. J. \& Combs, J. P. (2011). Data analysis in mixed research: A primer. International Journal of Education, 3(1), 1-25. https://www.doi.org/10.5296/ije.v3i1.618

Strömback, J. \& Kiousis, S. (2011). Political public relations: Defining and mapping an emergent field. En: J. Strömback \& S. Kiousis (Eds.): Political Public Relations. Principles and Applications (pp. 1-32). Routledge. https://www.doi.org/10.4324/9780203864173

Todorov, A.; Mandisodza, A. N.; Goren, A. \& Hall, C. C. (2005). Inferences of competence from faces predict election outcomes. Science, 308(5728), 1623-1626.

https://www.doi.org/10.1126/science.1110589

Van Aelst, P.; Sheafer, T. \& Stanyer, J. (2011). The personalization of mediated political communication: A review of concepts, operationalizations and key findings. Journalism, 13(2), 203-220. https://www.doi.org/10.1177/1464884911427802

Van Zuydam, S. \& Hendricks, S. F. (2018). Credibility enacted: Understanding the meaning of credible political leadership in the Dutch parliamentary election campaign of 2010. Journal of Political Marketing, 17(3), 258-281. https://www.doi.org/10.1080/15377857.2015.1039747

Walden, J. A.; Bortree, D. \& DiStaso, M. (2015). Reconsidering the public relations professionalblogger relationship: A coorientation study. Public Relations Review, 41(4), 526-532. https://www.doi.org/10.1016/j.pubrev.2015.06.002

Watanabe, K. (2017). Measuring news bias: Russia's official news agency ITAR-TASS' coverage of the Ukraine crisis. European Journal of Communication, 32(3), 224-241. https://www.doi.org/10.1177/0267323117695735

\subsection{Artículos relacionados}

Andstead, N. (2016). A different beast? Televised election debates in parliamentary democracies. The International Journal of Press/Politics, 21(4), 508-526. https://www.doi.org/10.1177/1940161216649953 
Fontenla Pedreira, J.; Conde Vázquez, E. y Máiz Bar, C. (2019). Uso de Twitter durante los debates electorales televisados en los comicios andaluces de 2018. Ámbitos. Revista Internacional de Comunicación, 46(2019), 213-240. https://www.doi.org/10.12795/Ambitos.2019.i46.12

García Marín, J. (2015). La cobertura mediática de los debates electorales en España. Revista Española de Ciencia Política, (38), 135-161. https://www.doi.org/10.1080/00028533.2001.11821554

Housholder, E. E. \& LaMarre, H. L. (2014). Facebook politics: Toward a process model for achieving political source credibility through social media. Journal of Information Technology \& Politics, 11(4), 368-382. https://www.doi.org/10.1080/19331681.2014.951753

Mazaira-Castro, A.; Rúas-Araújo, J. y Puentes-Rivera, I. (2019). Fact-checking en los debates electorales televisados de las elecciones generales de 2015 y 2016. Revista Latina de Comunicación Social, (74), 748-766. https://www.doi.org/10.4185/RLCS-2019-1355

Pressgrove, G. \& Kim, C. (2018). Stewardship, credibility and political communications: A content analysis of the 2016 election. Public Relations Review, 44(2), 247-255.

https://www.doi.org/10.1016/j.pubrev.2018.01.003

\section{AUTORES:}

\section{Alfredo Arceo Vacas}

Departamento de Teorías y Análisis de la Comunicación, Facultad de Ciencias de la Información, Universidad Complutense de Madrid (UCM). Profesor titular de la Universidad Complutense. Licenciado en Periodismo y Doctor en Ciencias de la Información por la misma universidad. Ha dirigido investigaciones sobre comunicación política, institucional y empresarial. Asimismo, coordinó la obra El portavoz en la comunicación de las organizaciones. Fundamentos teóricoprácticos, editada por el Servicio de Publicaciones de la Universidad de Alicante. Entre sus artículos, sobresale "The Identity of University Social Responsibility on the Websites of the Universities of the Autonomous Region of Madrid (Spain) and the State of Puebla (Mexico), As a Tool of Grassroots Public Diplomacy", en la revista American Behavioral Scientist. Fue director del Máster en Comunicación de las Organizaciones y, en la actualidad, del Máster Propio en Comunicación Corporativa y Publicitaria.

aarceo@ucm.es

Índice H: 6

Orcid ID: https://orcid.org/0000-0002-5981-4397

Google Scholar: https://scholar.google.es/citations?user=gBodfbUAAAAJ\&hl=es

Scopus: https://www.scopus.com/authid/detail.uri?authorId=6603281180

Academia.edu: https://independent.academia.edu/ArceoAlfredo

\section{Sergio Álvarez Sánchez}

Departamento de Teorías y Análisis de la Comunicación, Facultad de Ciencias de la Información, Universidad Complutense de Madrid (UCM). Investigador postdoctoral en el Departamento de Teorías y Análisis de la Comunicación de Universidad Complutense. Entre 2017 y 2019, fue investigador predoctoral en formación (convocatoria CT27/16-CT28/16 de los contratos SantanderUCM). Master en Comunicación de las Organizaciones y Doctor en Comunicación Audiovisual, Publicidad y Relaciones Públicas por la Universidad Complutense de Madrid. Ha realizado diversas investigaciones basadas en la teoría del framing, incluida su tesis doctoral: "Las Estrategias de 
Comunicación de los Actores Políticos Ante el Conflicto Social: Análisis de sus Marcos de Referencia para la Reforma Laboral de 2012". Acaba de publicar el artículo "Uso de una app móvil para evaluar la calidad de la enseñanza superior. Estudio de neuromarketing" (del que también es autor Alfredo Arceo) en Prisma Social.

sergioalvarezsanchez@ucm.es

Orcid ID: https://orcid.org/0000-0002-7494-8991

Google Scholar: https://scholar.google.com/citations?user=muJbJXkAAAAJ\&hl=en

ResearchGate: https://www.researchgate.net/profile/Sergio Alvarez34

Academia.edu: https://independent.academia.edu/SERGIOALVAREZSANCHEZ

\section{Olga Serrano Villalobos}

Departamento de Ciencias de la Comunicación y Sociología, Facultad de Ciencias de la Comunicación, Universidad Rey Juan Carlos (URJC). Profesora asociada de la Universidad Rey Juan Carlos y la Universidad Complutense, e investigadora de la cátedra de Comunicación y Salud del Departamento de Teorías y Análisis de la Comunicación de la UCM. Licenciada en Publicidad y Relaciones Públicas y Doctora en Ciencias de la Información por esta última institución. En la cátedra de Comunicación y Salud, ha investigado en la prevención a través de la imagen y las adicciones a nuevas tecnologías, tomando en consideración diferentes factores psicosociales. Uno de los principales resultados de este trabajo es el artículo "Tecno-adicción al sexo en la población juvenil: Propuesta del diseño de una escala de detección temprana", publicado en la Revista Comunicación y Salud en 2017.

olga.serrano@urjc.es

Índice H: 1

Orcid ID: https://orcid.org/0000-0002-8562-6680

Google Scholar: https://scholar.google.es/citations?user=Ibag1qAAAAAJ\&hl=es

ResearchGate: https://www.researchgate.net/profile/Olga Serrano2

Scopus: https://www.scopus.com/authid/detail.uri?authorId $=57200720869$

Academia.edu: https://independent.academia.edu/OLGASERRANOVILLALOBOS 